\title{
Imaging of Localized Surface Plasmon by Apertureless Scanning Near-Field Optical Microscopy
}

\author{
Soushi Ikeda, Yongfu Cai*, Qianwen Meng, Taichi Yokoyama, \\ Kenji Shinozaki, Takayuki Komatsu and Takayuki Ishibashi \\ Nagaoka University of Technology, 1603-1, Kamitomioka, Nagaoka, Niigata 940-2188, Japan
}

(Received February 16, 2015; accepted May 25, 2015)

Key words: surface plasmon polariton, apertureless scanning near-field optical microscopy, cross grooves

We measured the distribution of surface plasmon polaritons (SPPs) on cross grooves fabricated in Au film by apertureless scanning near-field optical microscopy (aSNOM). SNOM signals around horizontal and vertical grooves had double peaks and a single peak, respectively. To understand these different behaviors, we carried out simulations by the finite element method. From the simulation results, we found that the double peaks that appeared near the left and right edges of the horizontal groove were due to localized surface plasmons (LSPs). On the other hand, the strong signal that appeared near the center of the vertical groove was due to channel plasmon polariton. The SPP excitation depended on the direction of the electric field of the incident light and the cross sections of the grooves. This experiment reveals that aSNOM is a powerful tool for observing the distribution of SPPs on the nanoscale.

\section{Introduction}

In the field of photonics, surface plasmon polaritons (SPPs) are one of the most important physical phenomena. Because SPPs can enhance the electric field of incident light and confine the incident light onto nanoscale, it is used in many applications, such as biosensors, ${ }^{(1)}$ the trapping of polymer chains, ${ }^{(2)}$ and tip-enhanced Raman spectroscopy. ${ }^{(3)}$ Moreover, controlling polarization with SPPs has attracted much attention. Significant techniques that combine SPPs with nano-antennas to generate nanosized circularly polarized light have been reported, ${ }^{(4-6)}$ because nanosized circularly polarized light could be useful in significant phenomena, such as magnetization reversal using a 40 femtosecond circularly polarized laser pulse ${ }^{(7)}$ and spin excitation. ${ }^{(8)}$ Many techniques utilize localized surface plasmons (LSPs). Therefore, the analysis of the plasmon is very important, and techniques for SPP measurement are essential.

"Corresponding author: e-mail: yongfu_sai@mst.nagaokaut.ac.jp 
Apertureless scanning near-field optical microscopy (aSNOM) is one of the most suitable techniques for SPP measurement. ${ }^{(9)}$ An atomic force microscopy (AFM) cantilever is used to access the near field of the LSPs, and owing to the interaction between the cantilever and the near field, scattered light is generated, which provides information about the near field. We have developed an aSNOM with a high spatial resolution of $14 \mathrm{~nm},{ }^{(10)}$ and we have found that the polarization state of the scattered light is preserved. ${ }^{(1-13)}$ In this study, we report on SPP measurement in cross grooves made of Au by aSNOM with a high spatial resolution.

\section{Experiments}

\subsection{Fabrication of cross-groove sample}

In this study, we chose $\mathrm{Au}$, which has a plasmon resonance frequency in the visible light region, as a material for generating LSPs. The fabrication of cross-groove samples is shown in Fig. 1(a). A Au film was deposited by sputtering on a glass substrate $1 \mathrm{~cm} \times$

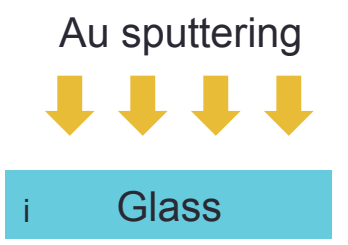

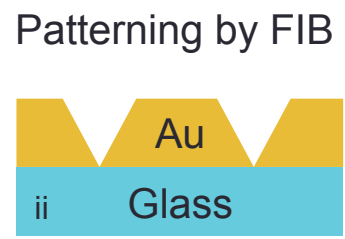

(a)

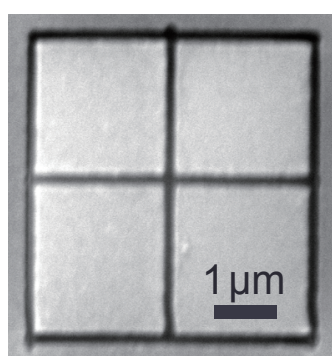

(b)

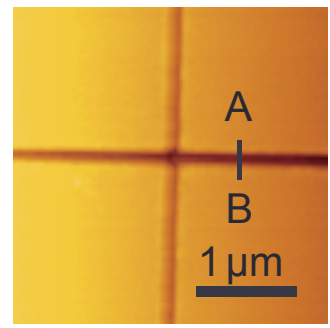

(c)

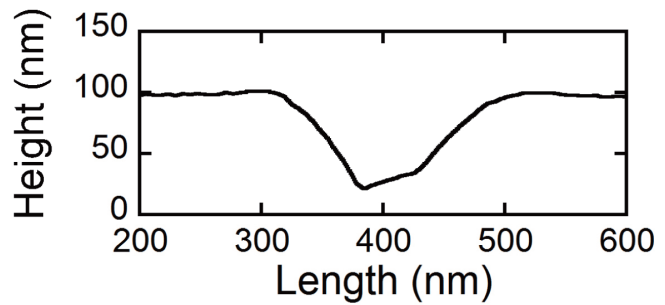

(d)

Fig. 1. (Color online) (a) Fabrication procedure of the cross-grooves sample. A Au film was deposited by sputtering on a glass substrate $(1 \mathrm{~cm} \times 1 \mathrm{~cm} \times 0.7 \mathrm{~mm}$ in size $)$, and the groove pattern was carved on the gold film using FIB. (b) SEM image of the whole cross-grooves sample. (c) Topographic image of the center of the sample. (d) Line profile of the horizontal groove from A to B in the topographic image. The height and width were approximately 80 and $100 \mathrm{~nm}$, respectively. 
$1 \mathrm{~cm} \times 0.7 \mathrm{~mm}$ in size [Fig. 1(a)]. The Au film thickness was estimated to be $70-80 \mathrm{~nm}$. A groove pattern was fabricated in the Au film using a focused ion beam (FIB) with a Ga ion dose of $2 \mathrm{nC} / \mu \mathrm{m}^{2}$. A SEM image of the whole cross-groove sample is shown in Fig. 1(b). The length of each groove is approximately $4 \mathrm{~mm}$. A topographic image of the center of the sample measured by AFM is shown in Fig. 1(c), and the line profile from A to B of the horizontal groove is shown in Fig. 1(d). We found that the height and width of the grooves were approximately 80 and $100 \mathrm{~nm}$, respectively.

\subsection{Setup of the aSNOM}

A schematic of the aSNOM is shown in Fig. 2. The aSNOM was based on a commercial AFM (Nanocute, Hitachi High-Tech Science, Japan). A cantilever (PRCDF40P, Olympus, Japan) made of Si with an extremity radius of $20 \mathrm{~nm}$ and a resonant frequency $\Omega$ of $443 \mathrm{kHz}$ was used. The AFM was operated in the dynamic force mode. A He-Ne laser (HN-410P, NEOARK, Japan) with a wavelength of $633 \mathrm{~nm}$ was focused by an objective lens (M Plan Apo $\times 10$, Mitutoyo, Japan) and impinged on the cantilever and the sample at an incident angle of approximately $90^{\circ}$. A polarizer was used to obtain linearly polarized light with a high extinction ratio. Scattered light passing through an analyzer was converted to an electric signal by a photomultiplier tube (PMT). Then, a lock-in amplifier (7280 DSP Lock-in Amplifier, Signal Recovery) was used to detect the signal at a third harmonic frequency $(3 \Omega)$. Finally, the signal from the lock-in amplifier was led to the scanning probe microscopy (SPM) station of the AFM. Consequently, the SNOM image and AFM image could be obtained simultaneously by scanning the cantilever on the sample.

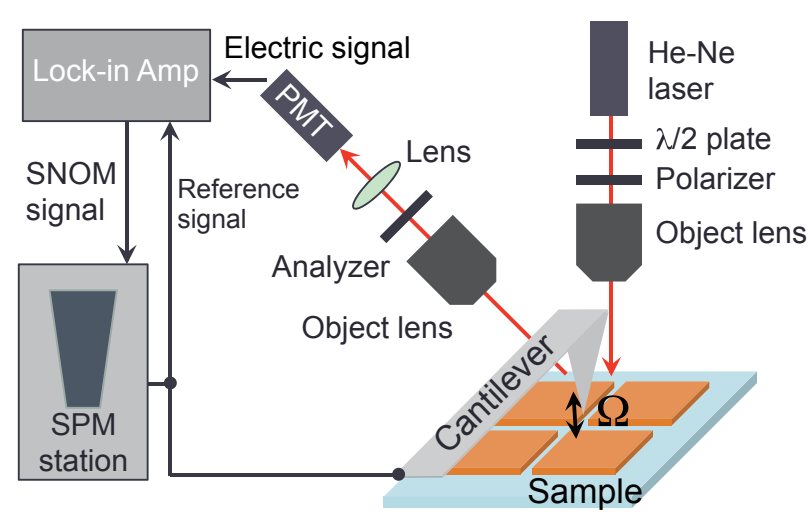

Fig. 2. (Color online) Schematic of the aSNOM based on a commercial AFM. The AFM was operated in dynamic force mode, and the resonant frequency $\Omega$ of the cantilever was $443 \mathrm{kHz}$. An incident light from a He-Ne laser impinged on the cantilever of the AFM and the sample. The scattered light from the near field was converted to an electric signal by the photomultiplier tube, and the near-field signal was extracted from the electric signal by the lock-in amplifier at a third harmonic frequency $3 \Omega$. 


\section{Results}

\subsection{SNOM images of cross-groove sample}

An AFM image of the cross-groove sample is shown in Fig. 3(a), and the SNOM image measured simultaneously without an analyzer is shown in Fig. 3(b). SNOM images measured with the analyzer's axis perpendicular (P-polarization) and parallel (S-polarization) to the axis of the polarizer are shown in Figs. 3(c) and 3(d), respectively.

In principle (i.e., without background noise signal), the brightness of the SNOM image corresponded to the intensity of the scattered light from the near field (i.e., intensity of electric field in near field). However, as shown in Figs. 3(b) and 3(d), strong periodical patterns were clearly observed, which were generated by the interference between the scattered light and the background light. On the other hand, we found

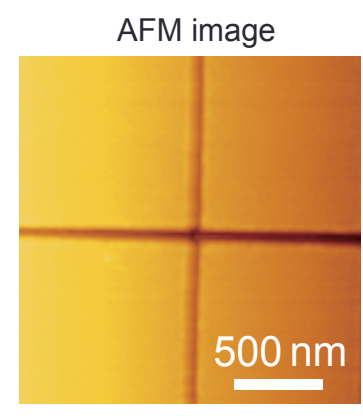

(a)

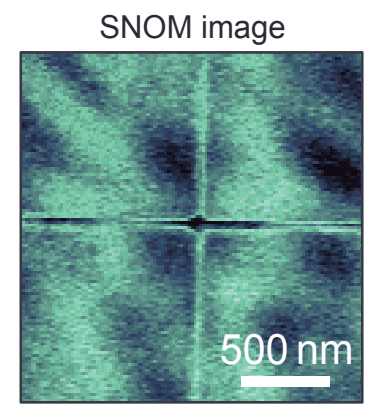

(b)

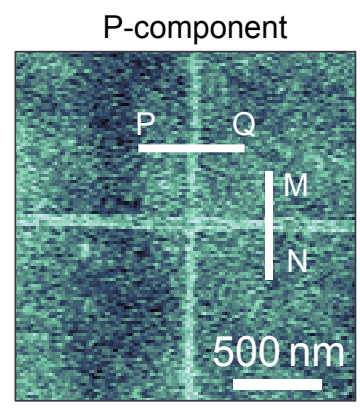

(c)

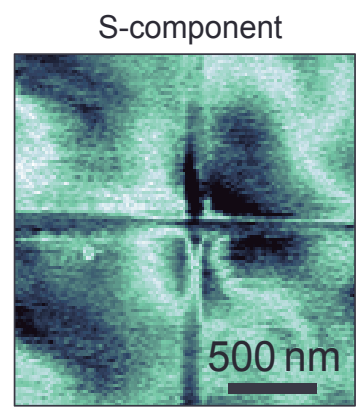

(d)

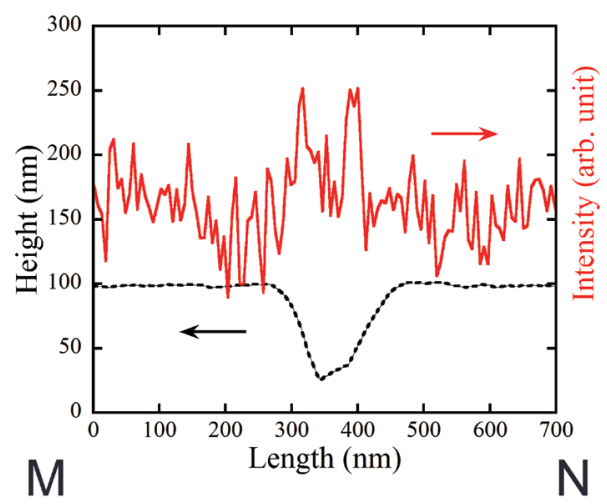

(e)

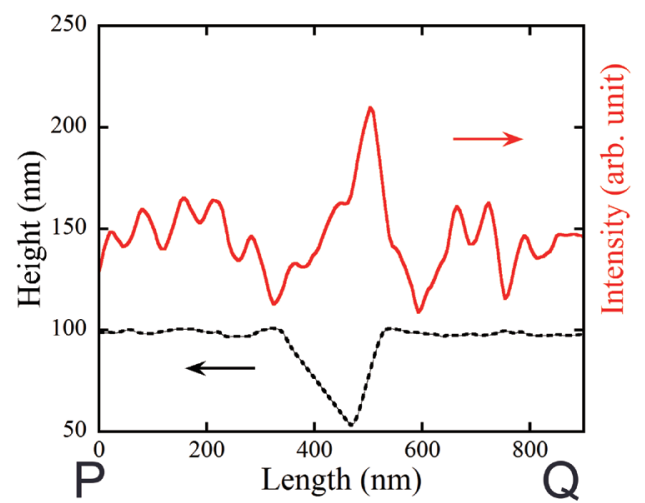

(f)

Fig. 3. (Color online) (a) AFM image and SNOM images measured, (b) without the analyzer, (c) for the P-component, and (d) for the S-component of the electric field of incident light. Line profiles along (e) MN and (f) PQ. 
that the effect of the background light was weak in the SNOM image measured for the P-component, as shown in Fig. 3(c). The polarization state of the background light was the same as that of the incident light (i.e., S-polarization); thus, most of the background light could be eliminated by a P-analyzer. This was the reason why the effect of the background light was weak. Therefore, we focused on the SNOM image measured with the P-component, because the information of SPPs could be clearly observed.

In Fig. 3(c), the difference in the signals around the grooves can be clearly observed, where the intensity becomes strong at the edges of the horizontal groove. On the other hand, the intensity becomes strong in the center of the vertical groove. Line profiles along the white lines of the horizontal and vertical grooves are shown in Figs. 3(e) and $3(\mathrm{f})$, respectively. The red lines represent the intensity of the SNOM signals and the black lines represent the height of grooves observed from the topographic image. From the line profiles, we found that two peaks appeared in the SNOM signal measured on the horizontal groove, and only one peak appeared in the vertical-groove measurement. Moreover, comparing the SNOM and AFM signals measured on the vertical groove shown in Fig. 3(f), we found a peak position shift (about $30 \mathrm{~nm}$ ) in the scanning direction of the cantilever. We suggested that this delay in the optical information was due to the scanning speed, because no similar delay was observed within the horizontal groove, whose long axis was parallel to the scan direction, as shown in Fig. 3(e).

\subsection{Simulation based on finite element method (FEM)}

To explain the experimental results, we carried out 2D simulation with the commercial software package Comsol Multiphysics 4.4 based on FEM. To reduce the size of the calculation domain, our model was smaller than the sample used in the experiment. The model consisted of an air layer on the top, a Au thin-film layer in the middle, and a glass substrate layer on the bottom. The parameters of the cross sections of the horizontal and vertical grooves from the AFM measurement were used in the simulation models. The incident light propagated perpendicularly with respect to the sample. The complex refractive index of Au is $0.201-\mathrm{i} 3.280$ at a wavelength of 633 $\mathrm{nm}$, and the dielectric constant is $-10.72+\mathrm{i} 1.32 .{ }^{(14)}$ A perfectly matched layer (PML) was used to eliminate unnecessary scattered light and reflected light. Note that the probe was not contained in the simulation.

The intensity distributions on the cross sections of the horizontal and vertical grooves are shown in Figs. 4(a) and 4(b), respectively. The intensity is given by

$$
\text { Intensity }=E_{x}^{2}+E_{y}{ }^{2}+E_{z}{ }^{2} \text {, }
$$

where $E_{x}, E_{y}$, and $E_{z}$ are the $x$-, $y$-, and $z$-direction components of the electric field, respectively. The white dotted lines indicate the contours of the cross section of Au. From the cross sections measured by AFM, we found that the depth of the horizontal groove was approximately $35 \mathrm{~nm}$ larger than that of the vertical groove; therefore, we considered that the Au film was separated into two parts in the horizontal groove, as shown in Fig. 4(a). In both cases, the electric field of the incident light was assumed to be perpendicular to the long axis of the grooves, although the dominant electric field 


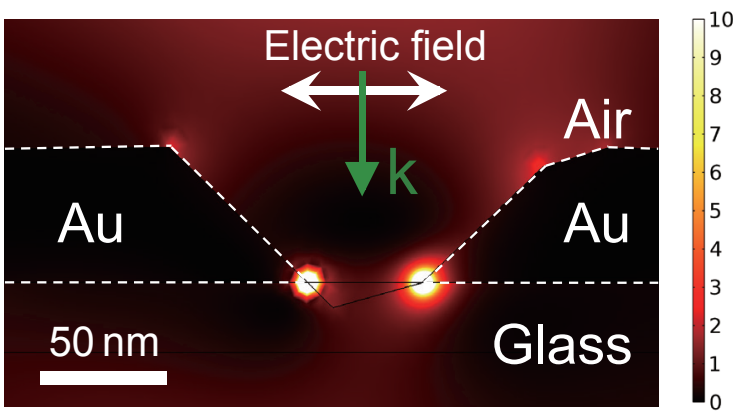

(a)

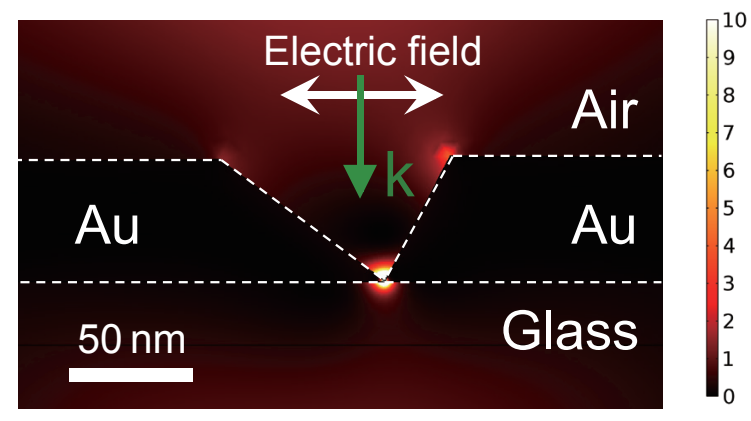

(b)

Fig. 4. (Color online) Distributions of intensity on the cross sections of the (a) horizontal and (b) vertical grooves calculated by simulation based on FEM. The white dotted lines indicate the contours of the cross section of Au.

of the incident light in the experiment was perpendicular to the long axis of the vertical groove and parallel to the long axis of the horizontal groove. The reason was that only the minor electric field that was perpendicular to the long axis could excite SPPs in the case of the horizontal groove. Two peaks appeared at the left and right edges of the horizontal groove, as shown in Fig. 4(a), which were due to the LSPs. On the other hand, one peak appeared at the bottom of the vertical groove, which was due to the channel surface plasmon. ${ }^{(6)}$ We considered that the simulation results agreed with the experimental results very well.

\section{Summary}

We have measured the distribution of SPPs on cross grooves fabricated in Au film. SNOM signals around horizontal and vertical grooves had double peaks and a single peak, respectively. To understand these different behaviors, we carried out simulations by FEM. From the simulation results, we found that the double peaks that appeared near the left and right edges of the horizontal groove were due to the LSPs. On the other hand, the strong signal that appeared near the center of the vertical groove was due to the SPPs. The SPP excitation depended on the direction of the electric field of the incident light and the cross sections of the grooves. This experiment showed the possibility that the SPPs could be measured at high resolution by the aSNOM.

\section{Acknowledgements}

This research was partly supported by the National Institute of Information and Communications Technology (NICT) and KAKENHI Grants-in-Aid for Scientific Research (B) (26286023). 


\section{References}

1 J. Homola: Anal. Bioanal. Chem. 377 (2003) 528.

2 M. Toshimitsu, Y. Matsumura, T. Shoji, N. Kitamura, M. Takase, K. Murakoshi, H. Yamauchi, S. Ito, H. Miyasaka, A. Nobuhiro, Y. Mizumoto, H. Ishihara and Y. Tsuboi: J. Phys. Chem. C 116 (2012) 14610.

3 W. Zhang, L. Huang, C. Santschi and O. J. F. Martin: Nano Lett. 10 (2012) 1006.

4 P. Biagioni, J. S. Huang, L. Duò, M. Finazzi and B. Hecht: Phys. Rev. Lett. 102 (2009) 256801.

5 K. Nakagawa, Y. Ashizawa, S. Ohnuki, A. Itoh and A. Tsukamoto: J. Appl. Phys. 109 (2011) $07 \mathrm{~B} 735$.

6 Y. Cai, S. Ikeda, K. Nakagawa, H. Kikuchi, N. Shimidzu and T. Ishibashi: Opt. Lett. 40 (2015) 1298.

7 C. D. Stanciu, F. Hansteen, A. V. Kimel, A. Kirilyuk, A. Tsukamoto, A. Itoh and T. Rasing: Phys. Rev. Lett. 99 (2007) 047601.

8 T. Satoh, Y. Terui, R. Moriya, B. A. Ivanov, K. Ando, E. Saitoh, T. Shimura and K. Kuroda: Nat. Photonics 6 (2012) 662.

9 R. Esteban, R. Vogelgesang, J. Dorfmuller, A. Dmitriev, C. Rockstuhl, C. Etrich and K. Kern: Nano Lett. 8 (2008) 3155.

10 M. Aoyagi, S. Niratisairak, T. Sioda and T. Ishibashi: IEEE Trans. Magn. 48 (2012) 3670.

11 Y. Cai, M. Aoyagi, A. Emoto, T. Shioda and T. Ishibashi: J. Magn. 18 (2013) 317.

12 Y. Cai, Q. Meng, A. Emoto, T. Shioda, H. Ono and T. Ishibashi: J. Magn. Soc. Jpn. 38 (2014) 127.

13 T. Ishibashi and Y. Cai: Nanoscale Res. Lett. 10 (2015) 375.

14 A. D. Rakić, A. B. Djurišić, J. M. Elazar and M. L. Majewski: Appl. Opt. 37 (1998) 5271. 